\title{
Three-dimensional plotted scaffolds with controlled pore size gradients: Effect of scaffold geometry on mechanical performance and cell seeding efficiency
}

\author{
Jorge M. Sobral, Sofia G. Caridade, Rui A. Sousa, João F. Mano*, Rui L. Reis \\ 3B's Research Group - Biomaterials, Biodegradables and Biomimetics, University of Minho, Headquarters of the European Institute of Excellence on Tissue \\ Engineering and Regenerative Medicine, AvePark, 4806-909 Taipas, Guimarães, Portugal \\ Institute for Biotechnology and Bioengineering, PT Associated Laboratory, Guimarães, Portugal
}

\section{A R T I C L E I N F O}

\section{Article history:}

Received 16 June 2010

Received in revised form 26 October 2010

Accepted 1 November 2010

Available online 4 November 2010

\section{Keywords:}

Tissue engineering

Regenerative medicine

Three-dimensional plotting

Porosity gradient

Seeding efficiency

\begin{abstract}
A B S T R A C T
Scaffolds produced by rapid prototyping (RP) techniques have proved their value for tissue engineering applications, due to their ability to produce predetermined forms and structures featuring fully interconnected pore architectures. Nevertheless, low cell seeding efficiency and non-uniform distribution of cells remain major limitations when using such types of scaffold. This can be mainly attributed to the inadequate pore architecture of scaffolds produced by RP and the limited efficiency of cell seeding techniques normally adopted. In this study we aimed at producing scaffolds with pore size gradients to enhance cell seeding efficiency and control the spatial organization of cells within the scaffold. Scaffolds based on blends of starch with poly( $\varepsilon$-caprolactone) featuring both homogeneously spaced pores (based on pore sizes of 0.75 and $0.1 \mathrm{~mm}$ ) and pore size gradients (based on pore sizes of $0.1-0.75-0.1$ and $0.75-0.1-$ $0.75 \mathrm{~mm}$ ) were designed and produced by three-dimensional plotting. The mechanical performance of the scaffolds was characterized using dynamic mechanical analysis (DMA) and conventional compression testing under wet conditions and subsequently characterized using scanning electron microscopy and micro-computed tomography. Osteoblast-like cells were seeded onto such scaffolds to investigate cell seeding efficiency and the ability to control the zonal distribution of cells upon seeding. Scaffolds featuring continuous pore size gradients were originally produced. These scaffolds were shown to have intermediate mechanical and morphological properties compared with homogenous pore size scaffolds. The pore size gradient scaffolds improved seeding efficiency from $\sim 35 \%$ in homogeneous scaffolds to $\sim 70 \%$ under static culture conditions. Fluorescence images of cross-sections of the scaffolds revealed that scaffolds with pore size gradients induce a more homogeneous distribution of cells within the scaffold.
\end{abstract}

(c) 2010 Acta Materialia Inc. Published by Elsevier Ltd. All rights reserved.

\section{Introduction}

Tissue engineering aims at restoring or regenerating a damaged tissue by combining cells with three-dimensional (3D) porous scaffolds. After isolation and eventual in vitro expansion, cells are seeded on 3D scaffolds and implanted directly or at a later stage in the patient [1]. Control of the cellular micro-architecture inside the scaffolds is of great importance when developing tissue engineering constructs. Moreover, early studies suggest that the micro-architecture of scaffolds might influence cell attachment and orientation and induce different biological behaviors [2-4]. Ultimately, optimizing and controlling these characteristics could lead to better implants when attempting to restore damaged tissues. Rapid prototyping (RP) is one of the most promising techniques

\footnotetext{
* Corresponding author. Address: 3B's Research Group - Biomaterials, Biodegradables and Biomimetics, AvePark, Zona Industrial da Gandra, S. Cláudio do Barco, 4806-909 Caldas das Taipas, Guimarães, Portugal. Tel.: +351 253510904; fax: +351 253510909.

E-mail address: jmano@dep.uminho.pt (J.F. Mano).
}

for designing and producing scaffolds for tissue engineering applications [5-11]. Many studies on the optimization of RP techniques and scaffolds fabricated by these techniques have been reported in the past few years [12-17]. The scaffolds are usually characterized by their $100 \%$ interconnected pores, fully computer controlled architecture and high porosities, which facilitate nutrient perfusion, essential to ensure cell viability. However, these techniques also present some drawbacks, including low resolution, which only allows fabrication of scaffolds with large pore sizes compared with the dimensions of a cell. This often leads to low cell seeding efficiencies (25-40\%) and to a non-uniform distribution of cells along the scaffolds [18]. From a tissue engineering point of view it is known that high cell densities are closely related to improved tissue formation in 3D scaffolds [19-23]. However, achieving a high cell seeding efficiency is very difficult, mainly due to the intrinsic scaffold characteristics (large pore size, poor cell-material adhesion and open pore architecture, among others) and limited cell seeding techniques [24]. The shortage of cells upon seeding requires longer periods of cell culture in order to obtain viable constructs. There is also some evidence that the growth of the cells 
in the scaffolds can be affected by the scaffold architecture due to a lack of ingress of nutrients and metabolic product removal [25]. Moreover, it is well established that an inhomogeneous distribution of the cells upon seeding is associated with low rates of tissue formation [19], a less uniform tissue [23] and a different cell differentiation behavior [22]. Therefore, a low cell seeding efficiency and heterogeneous distribution of cells in RP scaffolds are major drawbacks of these scaffolds in tissue engineering applications, which demands further research into the optimization of scaffold architectures and materials. One of the main aspects of scaffold architecture is the pore structure, which is determined by the size, size distribution, geometry and continuity of the individual pores within the scaffold. The pore structure plays an essential role in cell migration and adhesion, tissue formation, mechanical properties and nutrient diffusion, among other things. As mentioned before, RP might be used to easily control the scaffold architecture in order to obtain better results in terms of combining enhanced tissue growth with adequate mechanical properties [17,26-31]. To our knowledge there have been few experiments using gradient pore size or porosity 3D scaffolds to control the spatial organization of cells and increase the seeding efficiency for tissue engineering applications [32-39], probably due to considerable difficulties in both fabrication and correlating biological responses with scaffold architecture. Previous work by our group has shown that cell seeding is less efficient when the cell culture medium travels from the top of a scaffold through a porous structure having more direct paths for passage of the medium. In this study we hypothesize that scaffolds produced by a one-step fabrication process featuring two pore size gradients along one direction can improve cell seeding efficiency as well as the spatial distribution of the cells within the scaffolds compared with scaffolds featuring homogeneously spaced pores. More tortuous conduits inside the scaffold should increase the residence time of cells in the scaffolds and increase the likelihood of contact between the cells and the surface of the scaffold.

For these experiments we used a starch-based material starch/ poly( $\varepsilon$-caprolactone) blend (SPCL). Although SPCL can reduce cell proliferation rates [40], its low cytotoxicity in vitro [40,41], good biodegradability [42], and weak potential to stimulate an inflammatory response in vivo [43] make it a promising material for tissue regeneration applications. The improved scaffold architectures developed in this study are expected to further improve the mimicking of human tissue structures, as zonal variations in extracellular matrix are usually found in connective tissues.

\section{Materials and methods}

\section{1. $3 D$ scaffold fabrication}

The material studied in this work was a blend of corn starch with $\operatorname{poly}(\varepsilon$-caprolactone) (30/70 wt.\%, Novamont, Italy) as this material has previously been optimized for use in 3D scaffolds produced by 3D plotting and injection molding for tissue engineering applications [37,43]. SPCL granules were milled in an ultra-centrifugal mill (ZM-100, Retsch, Germany) with liquid nitrogen at 14,000 r.p.m. in order to facilitate melting during processing. The scaffolds used in all experiments were fabricated using 3D plotting (Bioplotter, EnvisionTec $\mathrm{GmbH}$, Germany). Alternating layers were oriented at $90^{\circ}$ to each other. The scaffolds were fabricated as $5 \times 5 \times 5 \mathrm{~mm}$ cubes. The size of the scaffolds was due to the low speed of the 3D plotting process, which makes the production of a significant number of larger scaffolds than the ones here difficult. Each layer was plotted using a layer thickness $(d 1)$ of $0.19 \mathrm{~mm}$. Fig. 1 shows the dimensional parameters of the scaffolds produced. In this study various scaffold architectures were produced: two homogeneous scaffolds exhibiting fiber spacings (d3) of 0.75 (Homog 1) and $0.1 \mathrm{~mm}$ (Homog 2) and two gradient scaffolds exhibiting distinct pore size variation with depth. The two gradient scaffolds were Grad 1, in which the pore size in the outer layers $(d 3=0.75 \mathrm{~mm})$ progressively decreased by $0.05 \mathrm{~mm}$ layer by layer resulting in a pore size of $0.1 \mathrm{~mm}$ in the middle. and Grad 2, in which the pore size in the outer layers $(d 3=0.1 \mathrm{~mm})$ progressively increased by $0.05 \mathrm{~mm}$ layer by layer resulting in a pore size of $0.75 \mathrm{~mm}$ in the middle. A schematic representation of these scaffolds is presented in Fig. 2.

\subsection{Scaffold characterization}

\subsubsection{Scanning electron microscopy}

Morphological characterization of the scaffolds was performed by scanning electron microscopy (SEM) (Nova NanoSEM 30, FEI, USA). All specimens were pre-coated with a conductive layer of sputtered gold. The micrographs were taken at an accelerating voltage of $15 \mathrm{keV}$ at different magnifications.

\subsubsection{Micro-computed tomography}

The architecture of the scaffolds was analyzed by micro-computed tomography ( $\mu-\mathrm{CT}$ ) using a desktop micro-CT scanner (SkyScan 1072, Belgium) at a voltage of $40 \mathrm{kV}$ and a current of $248 \mu \mathrm{A}$. Three scaffolds for each experimental condition were scanned at $7.53 \mu \mathrm{m} x y z$ resolution with an exposure time of $1792 \mathrm{~ms}$. Isotropic slice data were obtained by the system and reconstructed into two-dimensional (2D) $x y$ slice images. Slice images were subsequently compiled and analyzed to render 3D xyz images and to obtain quantitative architectural parameters. A $\mu$-CT analyzer and $\mu$ CT volume realistic 3D visualization software from SkyScan (Belgium) were used for image processing to reconstruct and create/ visualize 3D scaffold representations. All samples were reconstructed using a circular region of interest (ROI) with 160 slices. Identical threshold levels were defined for all the samples in order to eliminate image noise and to distinguish dense material regions from pore voids. Threshold levels were additionally inverted to obtain total porosity and to analyze pore morphology and interconnectivity. The -CT equipment was calibrated to provide accurate data at the length scale utilized. Taking into account the diameter of the fibres and the sizes of the pores this technique provides reliable data on the structural characteristics of the scaffolds.

\subsubsection{Static mechanical testing}

The mechanical properties were determined using a universal tensile testing machine (Instron 4505) in compression mode. Samples were previously hydrated in phosphate-buffered saline (PBS) for $24 \mathrm{~h}$ and tested at $37{ }^{\circ} \mathrm{C}$. A cross-head speed of $2 \mathrm{~mm} \mathrm{~min} \mathrm{~m}^{-1}$ was used until $80 \%$ deformation. The modulus $(E)$ was estimated from the initial slope of the stress-strain curve (linear part of the curve). Creep recovery measurements were performed at $37^{\circ} \mathrm{C}$ for $24 \mathrm{~h}$ in PBS. After the stress was removed the samples were allowed to recover for $24 \mathrm{~h}$. The values reported are the average of five specimens. The percentage recovery was calculated using the equation:

$\operatorname{Recovery}(\%)=\frac{\text { final scaffold height }(24 h)}{\text { initial scaffold height }(\text { before loading })} \times 100$

\subsubsection{Dynamic mechanical analysis}

Compression tests were carried out by dynamic mechanical analysis (DMA) (Tritec 2000B, Triton Technology, UK) in order to characterize the mechanical properties of scaffolds with varying morphologies. Prior to any measurements the scaffolds were immersed in PBS until equilibrium was reached (overnight). The measurements were carried out at $37^{\circ} \mathrm{C}$ under full immersion of the 


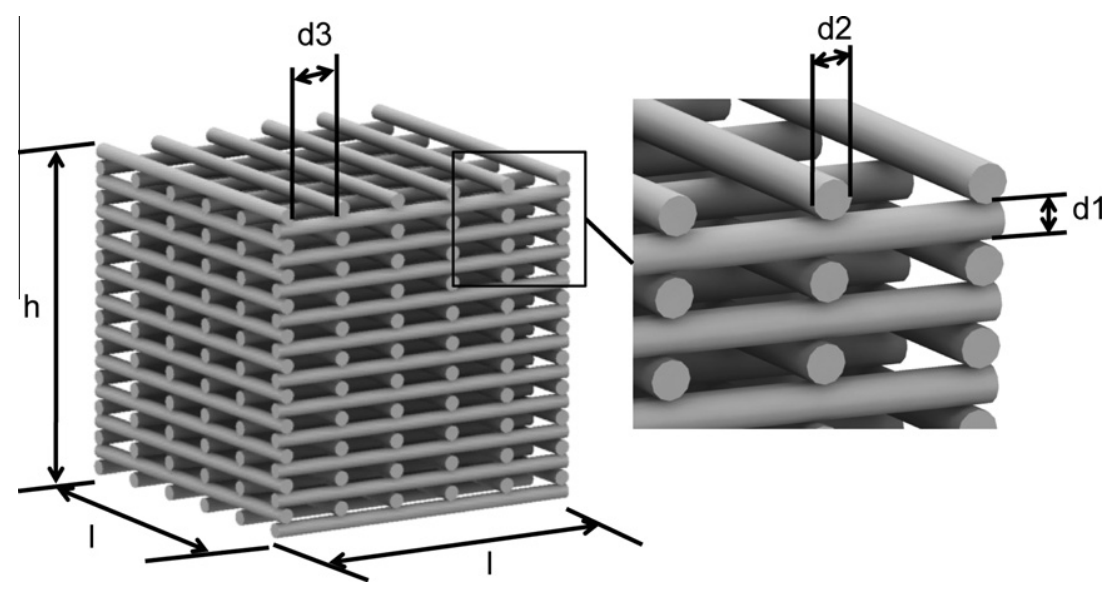

Fig. 1. Scaffold dimensional parameters: $d 3$, fiber spacing; $d 2$, fiber diameter; $d 1$, layer thickness; $l$, width of the scaffold; $h$, height of the scaffold.

\begin{tabular}{|c|c|c|c|c|}
\hline & $\begin{array}{l}\text { CAD 2D } \\
\text { Section }\end{array}$ & $\begin{array}{c}\text { Distance } \\
\text { Between Fibers }\end{array}$ & $\begin{array}{l}\text { CAD 3D } \\
\text { Models }\end{array}$ & $\begin{array}{c}\text { SEM } \\
\text { Pictures }\end{array}$ \\
\hline Grad 1 & 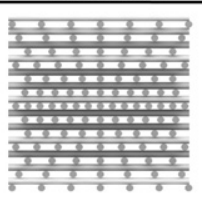 & $\begin{array}{c}\downarrow^{750 \mu \mathrm{m}} \\
100 \mu \mathrm{m} \\
\downarrow^{\downarrow} \mu \mathrm{mm}\end{array}$ & 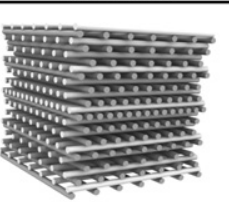 & 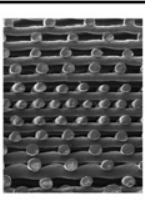 \\
\hline Grad 2 & 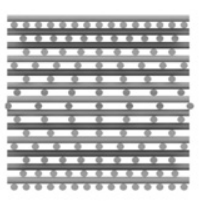 & 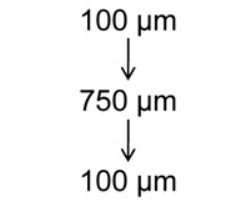 & 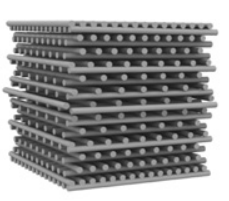 & \\
\hline Homog 1 & 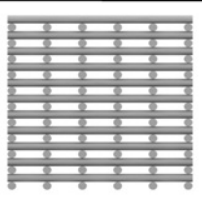 & $\left.\right|_{750 \mu \mathrm{m}} ^{750 \mu \mathrm{m}}$ & 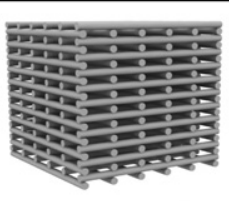 & 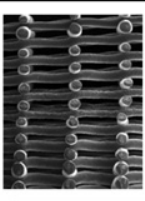 \\
\hline Homog 2 & 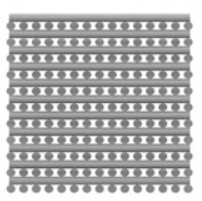 & $\left.\right|_{100 \mu \mathrm{m}} ^{100 \mu \mathrm{m}}$ & 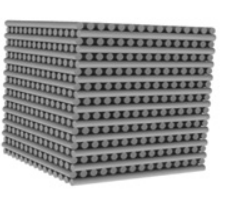 & 99 \\
\hline
\end{tabular}

Fig. 2. CAD 2D sections, pore size along the scaffolds, 3D models and SEM pictures for both homogeneous and pore size gradient scaffolds designed and produced by 3D plotting techniques for this study.

sample in PBS. The samples were cubs with sides of $5 \mathrm{~mm}$. Experiments were carried out in compression mode following cycles of increasing frequency ranging from 0.1 to $16 \mathrm{~Hz}$, with a constant strain amplitude of $0.07 \mathrm{~mm}$. The frequency range chosen covers the characteristic timescales of the periodic loads felt by the scaffold in vivo (e.g. frequency of skeletal movement and passage of blood, among others). The high frequency limit used in this study should provide information about the viscoelastic properties for the equivalent of short times (e.g. equivalent to a shock or sudden impact felt by the construct). Moreover, the frequency range used is within the typical frequency interval employed in DMA studies.

\subsection{Seeding efficiency and distribution of osteoblast-like cells}

\subsubsection{Cell culture}

The human osteosarcoma cell line SaOs-2 was obtained from the European Collection of Cell Cultures (ECCC, UK). Cells were cul- tured at $37{ }^{\circ} \mathrm{C}$ in a humidified atmosphere with $5 \% \mathrm{CO}_{2}$ using Dulbecco's modified Eagle's medium (Sigma, USA) supplemented with $10 \%$ heat-inactivated fetal bovine serum (FBS, Biochrom AG, Germany) and $1 \%$ antibiotic (Gibco, USA). A third passage SaOs- 2 cell suspension $\left(1 \times 10^{6}\right.$ cells) was prepared by trypsinisation $(0.25 \%$ trypsin/EDTA solution, Sigma, USA). The scaffolds were placed in 48-well plates not treated for tissue culture to diminish adhesion and proliferation of cells on the surface of the wells. A drop of $50 \mu \mathrm{l}$ of the cell suspension was seeded on the top surface of each scaffold. After a period of $3 \mathrm{~h} 1 \mathrm{ml}$ of medium was additionally added to each well. A similar technique has been used elsewhere [44]. The scaffolds were left under standard cell culture conditions for $12 \mathrm{~h}$ to give the cells enough time to adhere to and establish themselves in the different scaffolds. Due to the technology employed in this work the resulting scaffolds exhibit full interconnectivity between the pores and therefore one would expect not only that cells have access to nutrients and oxygen in the first $12 \mathrm{~h}$ but 
also that cell death in the interior of the scaffolds is not an important factor that could affect cell seeding efficiency and distribution.

\subsubsection{Fluorescence analysis}

All scaffolds selected for fluorescence microscopy were fixed in $2.5 \%$ glutaraldehyde (Sigma, USA) solution in PBS for $1 \mathrm{~h}$ at $4{ }^{\circ} \mathrm{C}$. After washing in PBS the specimens were cut using a bistoury. The sections were stained with the nuclear stain DAPI (Sigma) for $1 \mathrm{~min}$ and then washed three times with PBS. The sections were observed via fluorescence microscopy with a UV filter for DAPI staining. The images were also analyzed using Image with a color histogram plugin. A threshold in the blue range was applied to the images in order to remove the background and display only the blue associated with staining of the cells. The images were then divided into eight slices from top to bottom and the percentage of blue against the black was calculated for each slice using ImageJ.

\subsubsection{Seeding efficiency analysis}

In order to determine seeding efficiency scaffolds were kept for $12 \mathrm{~h}$ under static conditions following cell seeding. Cells were left in the scaffolds for $12 \mathrm{~h}$ to guarantee that they had enough time to attach and adapt to the different scaffold architectures analyzed in this work. After this period the scaffolds were removed and the remaining cells in the wells were counted using a counting chamber method. Upon removal of the medium, cells attached to the bottom of the wells were removed by trypsinisation ( $0.25 \%$ trypsin/EDTA solution, Sigma, USA). The seeding efficiency for each scaffold was calculated by taking into account the initial number of cells that were added to the scaffold and the residual number of cells in the respective well after $12 \mathrm{~h}$. One would expect very low proliferation of cells in the non-tissue culture treated wells during the initial $12 \mathrm{~h}$ and the proliferation rate in the well to be constant, independent of the scaffold type in the well. Therefore, the contribution of cell proliferation in the well to absolute seeding efficiency values during the $12 \mathrm{~h}$ period was ignored. Moreover, this technique can provide a valid comparison between the seeding efficiency on different scaffolds using the same seeding technique and materials, since any significant differences in seeding efficiency can only be due to the different type of scaffold. This technique was also chosen in order to be able to perform a complete of analysis at different time points using the same samples (cell distribution by fluorescence analysis and scanning electron microscopy, among others). The values reported are the averages for at least 10 specimens of each type of scaffold. The seeding efficiency was calculated using the equation:

$$
\begin{aligned}
\text { Seeding efficiency }(\%)= & \frac{(\text { cells added to scaffold }- \text { cells in wells })}{\text { cells added to scaffold }} \\
& \times 100
\end{aligned}
$$

\subsection{Statistical analysis}

Eight replicates were used for all the analyses. The data were statistically analyzed using a $t$-test. In all statistical comparisons $P<0.05$ was considered statistically significant.

\section{Results and discussion}

\subsection{Scaffold characterization}

\subsubsection{Scaffold morphology and architecture}

In the present study we have evaluated the effect of scaffold architecture on mechanical performance, seeding efficiency and cell distribution. We were successful in producing scaffolds with two pore size gradients, as well as homogeneous scaffolds with different pore sizes (Fig. 3). The gradient pore size scaffolds were created by controlling the pore size parameters for each layer. Homogeneous scaffolds have no offset fiber distance between consecutive layers (Fig. 3), however, the gradient scaffolds have a continuous offset $(0.05 \mathrm{~mm})$ between consecutive layers. Fig. 4 shows the junction between orthogonal fibers and final fiber surface. The
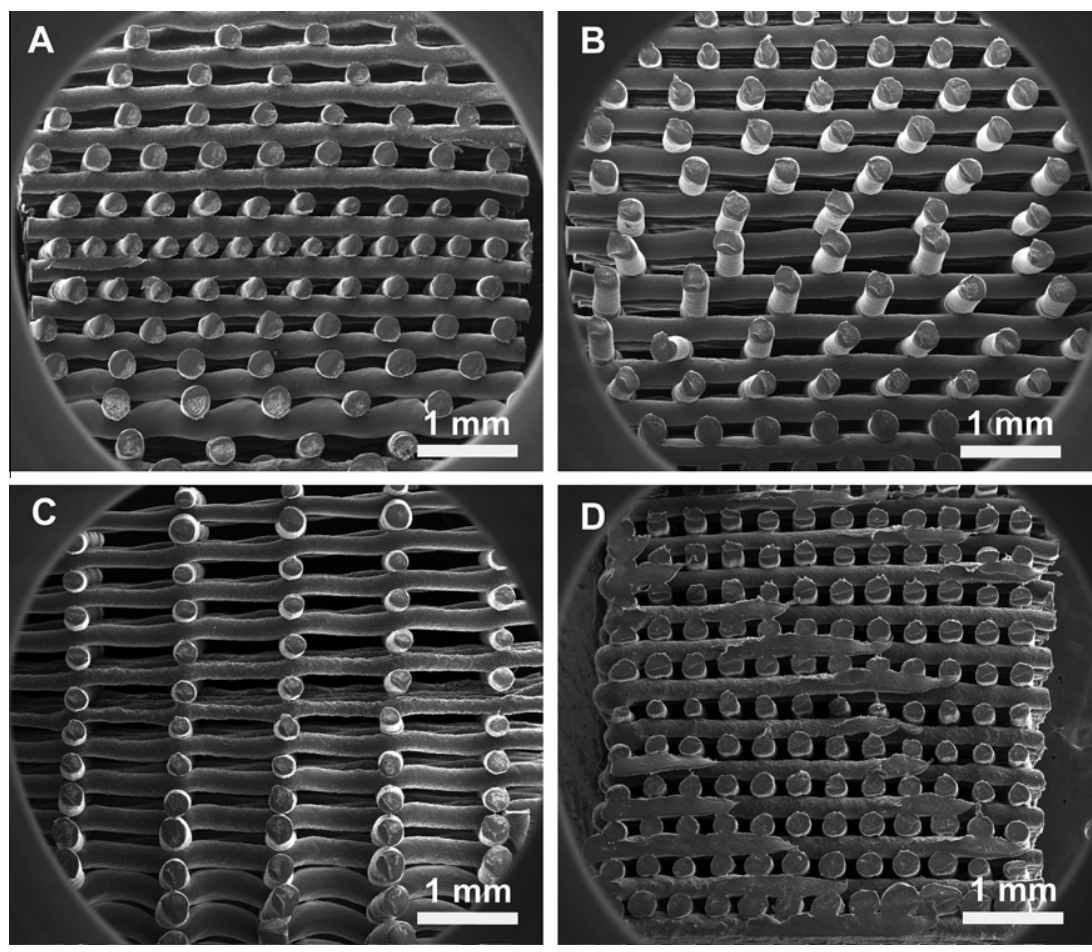

Fig. 3. SEM pictures of cross-sections of (A) Grad 1, (B) Grad 2, (C) Homog 1 and (D) homog 2 scaffolds. 

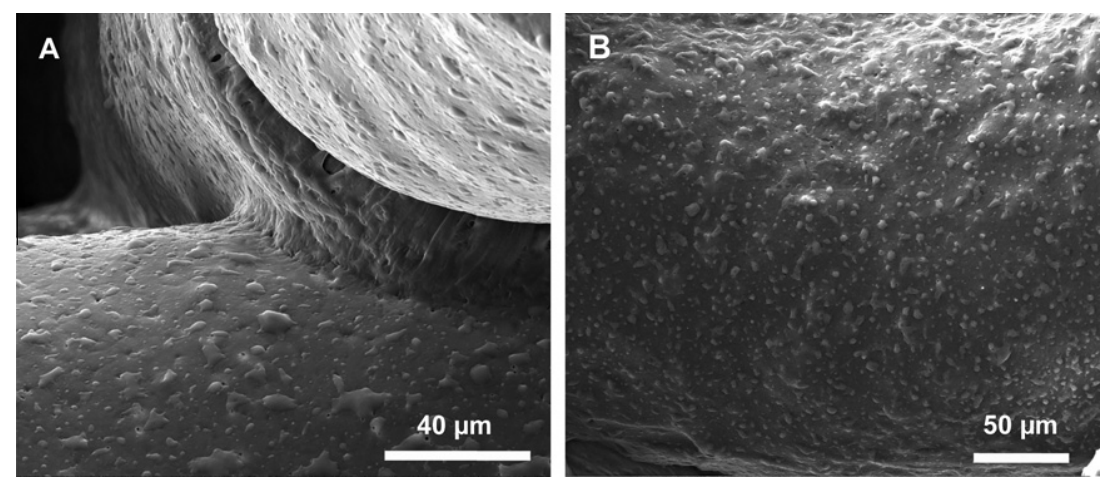

Fig. 4. SEM pictures of (A) the junction between fibres and (B) the fiber surface.

design parameters adopted, in terms of the distance between layers $(d 1=0.19 \mathrm{~mm})$, achieved very good bonding between the fibers, which is essential for mechanical stability of the scaffolds $[45,46]$. This is consistent with the lack of scaffold delamination upon extensive manipulation and subsequent mechanical testing. Fig. 4a and 4b also clearly shows the surface of the fibres: one can detect some asperities randomly dispersed on the surface with dimensions of about 5-10 $\mu \mathrm{m}$. Although we have not analyzed the suitability of the surface of SPCL plotted fibers for cell attachment, the micro-roughness developed upon processing is believed to play an important role in cell attachment and proliferation [2,3,47-49].

To our knowledge there are few studies that have reported a continuous layer by layer pore size gradient prepared by different techniques [33,50-52], and none using 3D plotting. In this work we fabricated scaffolds with a homogeneous pore size and others with porosity gradients. Fig. 5a and $5 \mathrm{~b}$ presents pore size and local porosity as a function of scaffold height as measured by SEM and $\mu-C T$ of five samples. Standard deviations are not represented in order to increase the readability of the figures. However, the error was below $0.015 \mathrm{~mm}$ for fiber spacing and $2.6 \%$ for porosity, which
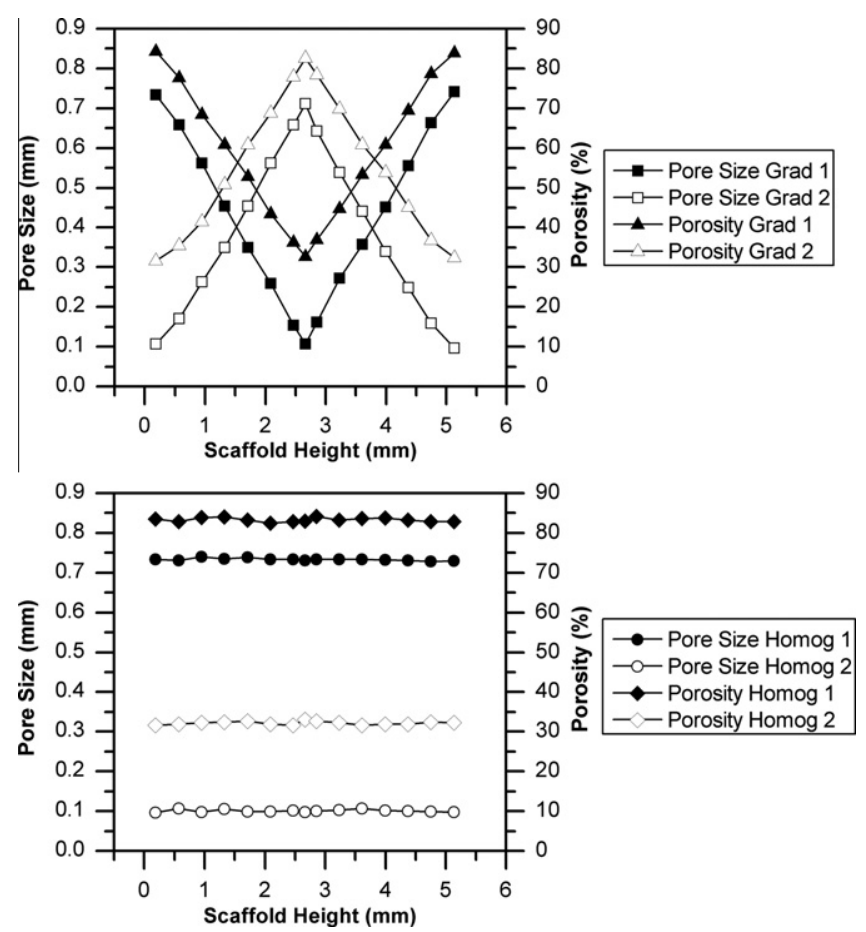

Fig. 5. Variations in porosity and fiber spacing of gradient and homogeneous scaffolds. testifies to the high reproducibility of scaffold fabrication. For homogeneous scaffolds the porosities for pore sizes of 0.75 and $0.1 \mathrm{~mm}$ are $84 \%$ and $31 \%$, respectively. In contrast, for gradient scaffolds the porosity values vary continuously between $84 \%$ and $31 \%$.

In order to fully understand the mechanical properties and the cell seeding efficiency of scaffolds we determined the total porosity of each type of construct by $\mu$-CT (Fig. 6). As expected, for gradient scaffolds the total porosity values are intermediate between those obtained for homogeneous scaffolds. For Grad 1 the total porosity is $60 \%$ while for Grad 2 the total porosity is $56 \%$. This is explained by the fact that Grad 2 scaffolds have two layers with the lowest pore size $(d 3=0.1 \mathrm{~mm})$, while Grad 1 scaffolds have only one layer with this pore size present at the middle of the scaffold. The Homog 1 scaffolds have a porosity of $81 \%$, while the Homog 2 scaffolds have a porosity of $31 \%$, which correlates with the values in

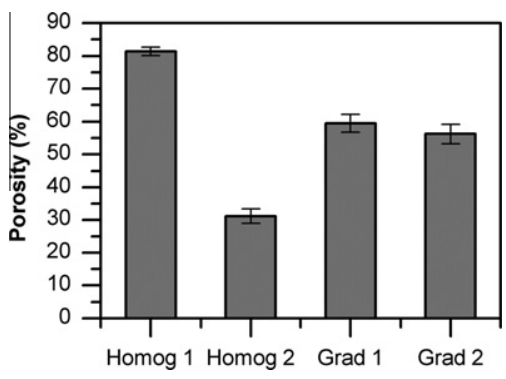

Fig. 6. Total porosity in the scaffolds studied.

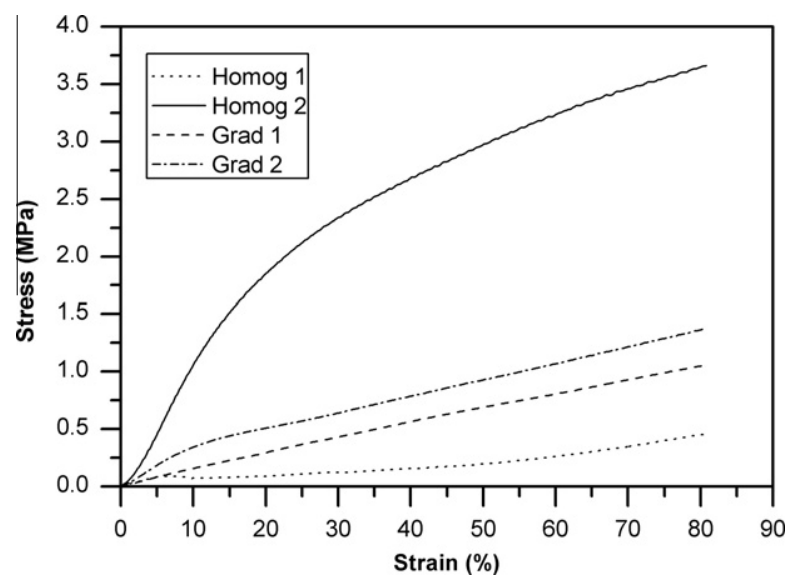

Fig. 7. Representative stress-strain curves for each type of scaffold 
previous studies using similar types of scaffolds and similar fabrication techniques $[16,26,30,31]$.

\subsubsection{Mechanical analysis}

Characterization of the compressive characteristics of the scaffolds is important in many tissue engineering applications [53]. Fig. 7 shows the stress and strain curves obtained for all samples at $37 \mathrm{C}$. There is a clear difference in the energy absorption upon deformation between scaffolds up to $80 \%$ strain (see Fig. 7). When comparing the results presented in Fig. 7 with those in Fig. 8a it is evident that gradient scaffolds have intermediate mechanical performance compared with homogeneous scaffolds, indicating the expected relationship between porosity of the scaffolds and the respective mechanical properties. Nevertheless, this correlation is not linear, as the relative difference in porosity between the Grad $1 /$ Grad 2 and Homog 2 scaffolds does not on its own account for the differences in stiffness. This may be attributed to other factors, such as the orientation and relative positioning of the fibers along the scaffold, which can play a key role in determining the final mechanical performance [26,54-56]. A starch-polycaprolactone (30/70 wt.\%) material has been shown to have a Young's modulus of approximately $400 \mathrm{MPa}$. However, these values may vary considerably depending the on temperature and humidity (from 100 to $800 \mathrm{MPa}$ ) [57,58]. Moreover, when considering scaffolds with high porosities tested under physiological conditions the Young's modulus can decrease to $0.8-30 \mathrm{MPa}$ [59]. The values reported here correlate with those found in the literature for 3D scaffolds based on starch [60].

Scaffold resilience upon creep deformation is very important in tissue engineering applications [53]. Fig. 8b shows that the architecture of a scaffold influences its resilience. The gradient scaffolds recovered approximately $77 \%$ of their initial height after a deformation of $60 \%$, while the homogeneous scaffolds Homog 1 and
Homog 2 recovered $87 \%$ and $67 \%$ of their initial height, respectively. The total porosity of the scaffolds can once more be related to these values. It is known that the modulus of interconnected porous materials is dependent on their relative density, i.e. the inverse of porosity. This is evident from the greater stiffness of Homog 2 compared with the more porous architectures produced here. The Gibson and Ashby model for the compression of open cell elastic foams shows that upon continuous compression buckling of the structure will ultimately occur at a given collapse stress that is proportional to the material density. Although such a model is not directly related to our case, the basic fundamentals are still useful in explaining the different resilience values reported for the different scaffold architectures. For a given compression deformation applied during creep testing a higher stress is imposed on Homog 2 scaffolds, due to its greater stiffness, compared with the Grad 1, Grad 2 and Homog 1 scaffolds. Therefore, for the same imposed deformation the higher stress applied to Homog 2 scaffolds causes more extensive collapse of the structure. These results indicate that despite stiffness being a parameter that needs to be considered in the design of new scaffolds for biomedical applications it can negatively affect the ability of a scaffold to recover after deformation. One could argue that the ability of a scaffold to avoid plastic deformation, remaining in an elastic state, and being able to recover can be as important as stiffness in the design of materials and scaffold architectures for biomedical applications.

The viscoelastic properties of a polymer determine the mechanical behavior when used as an implanted biomaterial. It has previously been shown that DMA is able to characterize the viscoelastic properties of starch-based biomaterials [61-63], the mechanical responses of which are highly dependent on the hydration level $[64,65]$. DMA was used in this work to characterize the viscoelastic properties of the SPCL 3D scaffolds under wet conditions. The dependence of the storage modulus $\left(E^{\prime}\right)$ and frequency loss factor
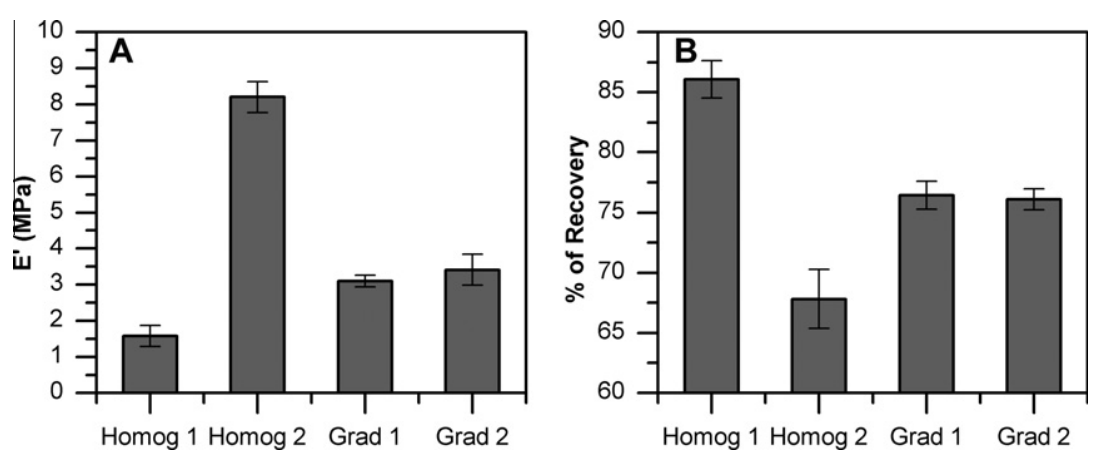

Fig. 8. Values of (A) Young's modulus and (B) percentage recovery for the scaffolds studied after a deformation of $80 \%$.
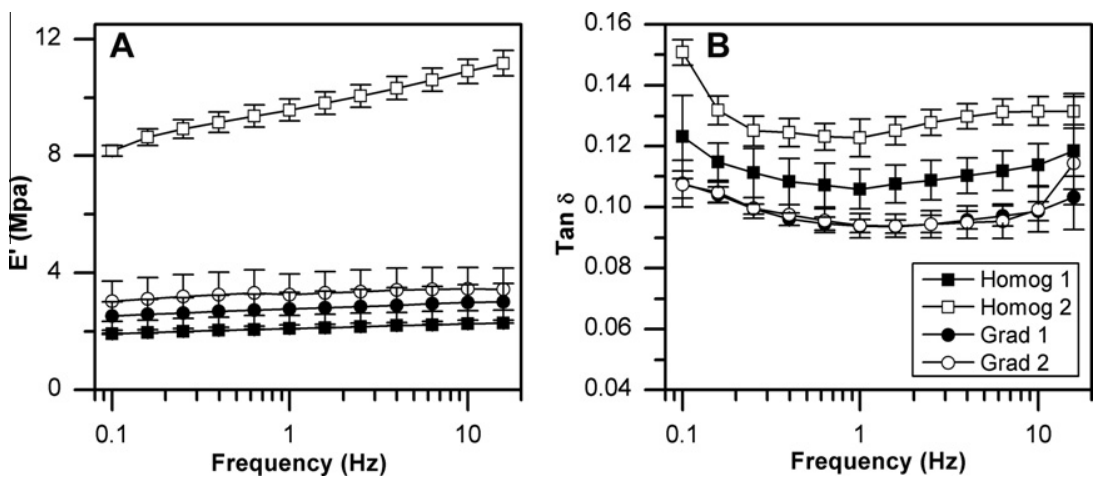

Fig. 9. Variations in (A) $E^{\prime}$ and (B) $\tan \delta$ with frequency for the scaffolds studied at $37^{\circ} \mathrm{C}$ in $\mathrm{PBS}$. 


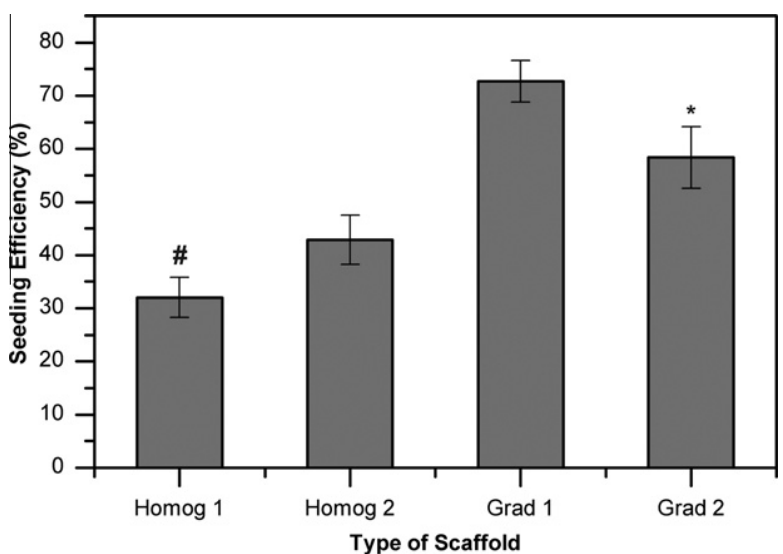

Fig. 10. Seeding efficiency for each type of scaffold. "Statistical significance ( $t$-test, $P<0.05)$ in gradient scaffolds. "Statistical significance $(t$-test, $P<0.05)$ in homogeneous scaffolds.

$\left(\tan \delta\right.$ ) at $37^{\circ} \mathrm{C}$ in PBS solution are presented in Fig. 9. Essentially, a slight increase in $E^{\prime}$ and decrease in $\tan \delta$ is detected for SPCL with an increase in frequency in the range analyzed $(0.1$ and $16 \mathrm{~Hz})$. $\tan \delta$ is a measure of the damping capability of the material and is related to the relative weight of the viscous and elastic features of the material and is not directly linked to porosity. Therefore, the trends in $\tan \delta$ among the different structures are not the same as observed for the Young's modulus and elastic modulus, which are strongly dependent on porosity and scaffold internal architecture. Fig. 9 also shows that the values of $\tan \delta$ at body temperature were between 0.09 and 0.15 , which is a narrow range for this type of test. These values are in agreement with those previously reported for this material [66-68] and indicate that SPCL is able to dissipate a significant fraction of the imposed mechanical energy. It would also be interesting to compare the obtained values of $\tan \delta$ with those found in native tissues. Other studies have shown that $\tan \delta$ values for biological tissues like bone are of the same order of magnitude as the values of $\tan \delta$ reported here [69]. Fig. 9b indicates that the structures with a gradient morphology have slightly higher $\tan \delta$ values than the homogeneous ones, but the differences observed between these two kinds of scaffolds are unclear and any discussion at this stage would be speculative. Regarding $E^{\prime}$, the values from DMA are very similar to those obtained under quasi-static testing, and the same relationships to porosity and pore architecture are again observed.

\subsection{Biological performance of the scaffolds}

\subsubsection{Seeding efficiency and distribution of cells}

To evaluate cell response to the scaffold architecture the constructs were cultured in vitro for $12 \mathrm{~h}$, after which all experiments were conducted. As was mentioned before, seeding efficiency plays a major role in tissue engineering approaches and is mainly affected by the scaffold material and the respective porous architecture. Fig. 10 shows the seeding efficiency for each scaffold type. The seeding efficiencies of Grad 1 and Grad 2 were approximately $70 \%$ and 56\%, respectively. For the homogeneous scaffolds Homog 1 and Homog 2 the seeding efficiencies were approximately $30 \%$ and $40 \%$, respectively, which correlates with the values in the literature $[18,27]$. The results indicate that in the gradient scaffolds, especially Grad 1, there was a significant improvement in seeding efficiency. Although seeding efficiency has been associated with the surface area available for cells to attach to, recent studies have shown that in RP scaffolds the pore structure and scaffold architecture can play a more important role $[31,70]$. The pore structure dictates the interaction of the scaffold and transplanted cells with the host tissue. As is known, only scaffolds having an interconnected porous structure are able to allow free bulk transport of biomolecules, and in scaffolds with large enough pores $(d>10 \mu \mathrm{m})$ cells are also able to migrate through them [71]. For instance, for bone in-growth [72] the minimum pore size for cell migration and nutrient diffusion is considered to be around $100 \mu \mathrm{m}$. However, pore sizes above $300 \mu \mathrm{m}$ are recommended to promote new bone formation and tissue vascularization. In addition, it is also known that 3D matrices with similar porosities but different pore geometries, such as fibrous versus spherical pores, can yield different mass transport profiles and thus differently influence cell behavior $[6,73]$. Therefore, the differences in cell seeding efficiency observed between the Grad 1 and Grad 2 scaffolds (with similar surface areas) and between the gradient scaffolds and Homog 2 (with a much higher surface area) can only be explained by the differences in scaffold architecture.

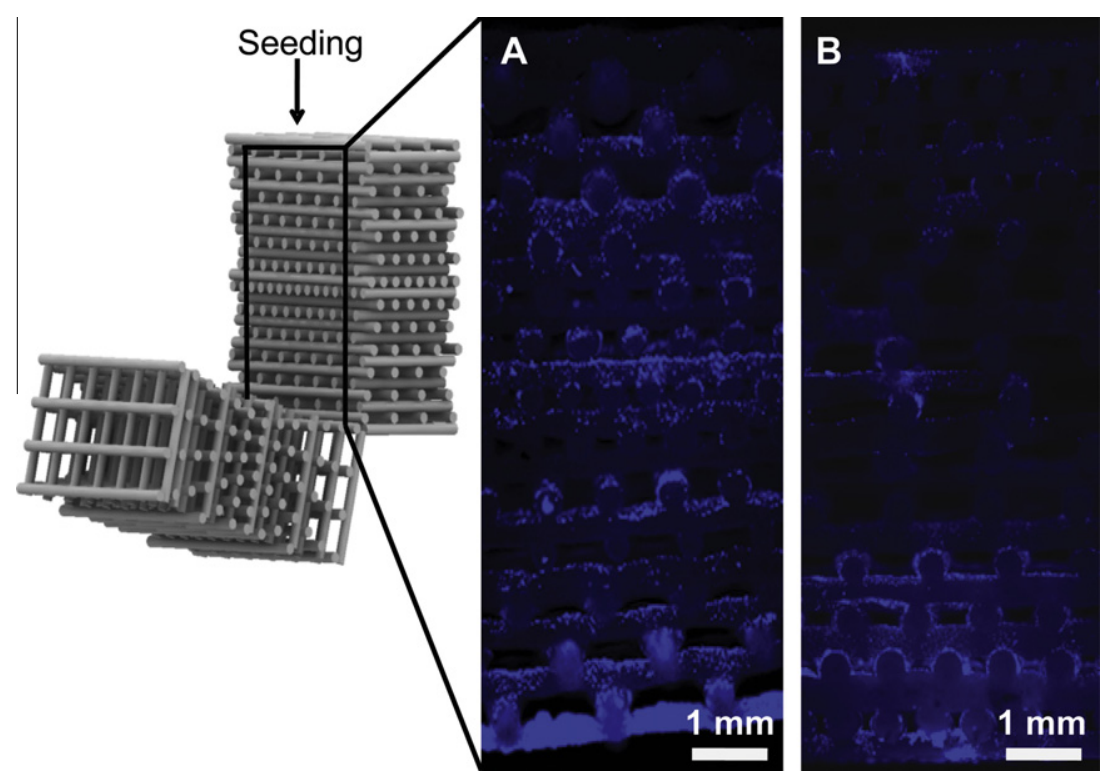

Fig. 11. Images taken with a florescence microscope of cross-sections of (A) Grad 1 and (B) Grad 2 scaffolds stained with DAPI. 


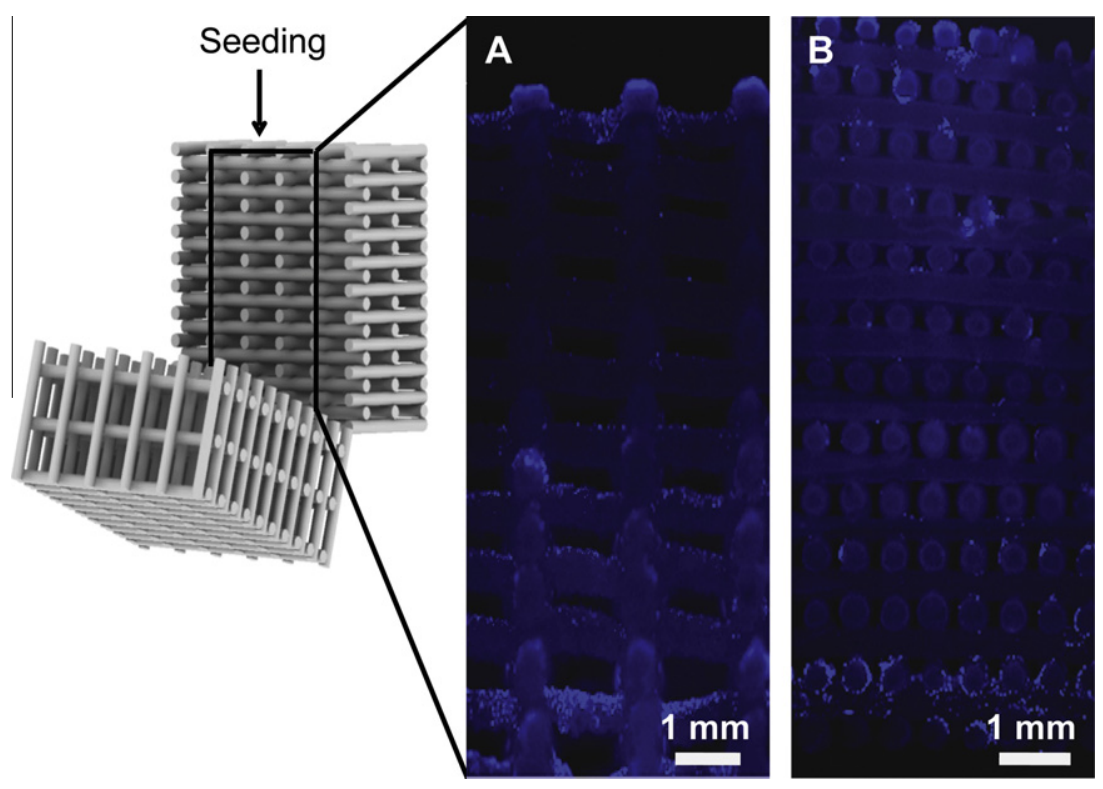

Fig. 12. Images taken with a florescence microscope of cross-sections of (A) Homog 1 and (B) Homog 2 scaffolds stained with DAPI.

The difference between the Homog 1 and Homog 2 scaffolds can be attributed to a higher surface area for the Homog 2 scaffold, which allows the adhesion of more cells to the top and lower layers. In addition, the higher structural density of the Homog 2 scaffolds arising from the higher number of junction sites between orthogonal fibres also contributes to an increased cell seeding efficiency. The existence of more junction points should be related to better anchorage of cells to the scaffolds, increasing the chances of cell adhesion upon seeding. With regard to the differences between the gradient and homogeneous scaffolds, the increase in seeding efficiency can be explained by the presence of an offset between consecutive layers in the gradient scaffolds $[12,26,27]$. However, the existence of a geometrical offset does not explain the absolute cell seeding efficiency nor the relative difference between the Grad 1 and Grad 2 scaffolds, as both have an offset. We believe that cell seeding efficiency is influenced by both the offset of consecutive scaffold layers and the flow conditions throughout the scaffold during cell seeding. The flow conditions when pipetting a cell suspension onto the scaffold resemble the flow under dynamic seeding $[74,75]$. A low flow rate within
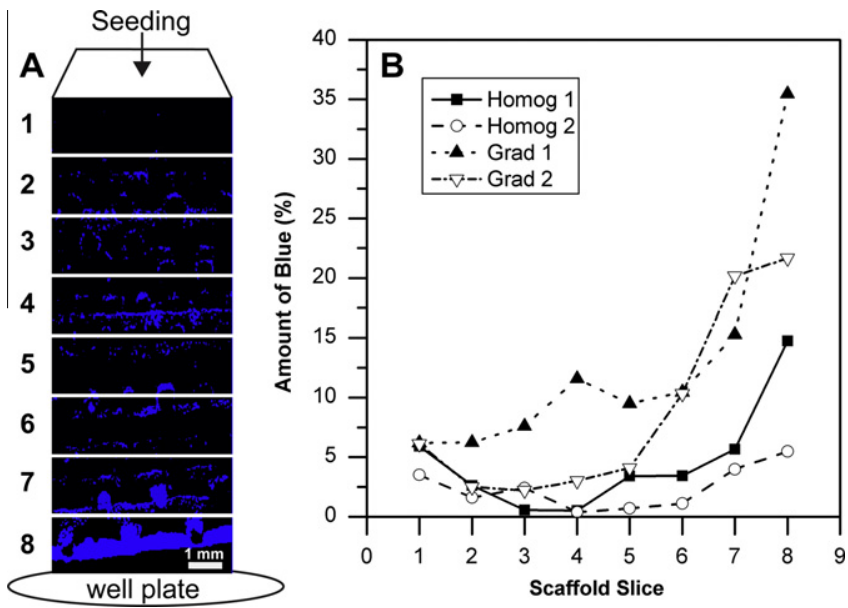

Fig. 13. Images showing (A) an example of a cross-section of scaffold Grad 1 cut into eight slices and filtered to show only the blue associated with the cell staining and (B) variation in the amount of blue in the scaffold slices from the top to the bottom. the scaffold upon seeding favors cell attachment at the fiber surface and junction sites, while high flow rates promote cell deposition at the bottom of the well. In addition, the existence of an offset or a complex pore shape can also enhance the average flow path of medium within the scaffold upon seeding, which also favors seeding efficiency. Thus, the flow will probably be conditioned by the size of the pores and the scaffold architecture. Figs. 11-13 show the cell distribution with height in the scaffolds. The results show that the distribution of cells is more homogeneous in Grad 1 scaffolds and in both gradient scaffolds compared with the homogeneous ones. The explanation for this might also be related to that given for the higher cell seeding efficiencies in the gradient scaffolds. The flow mechanics can probably explain why the cell distribution in Grad 1 (Figs. 11a and 13) is more homogeneous than in Grad 2 (Figs. 11b and 13). In Grad 1 scaffolds there is a decrease in pore size from the initial layer to the middle of the scaffold, which constrains flow through the scaffold. This, combined with the offset of the fibers favors cell seeding. In Grad 2 cells tend to become entrapped only in the final layers near the bottom of the well, due to the final decrease in pore size. The possibility that the seeding technique used might contribute to an asymmetric distribution of cells within the scaffolds (e.g. a tendency for cells to attach more to the bottom and top layers of the scaffolds) and that the same effect would not be observed using a different seeding technique (e.g. dynamic seeding with spinning flasks) cannot be ruled out. However, the observation that cell distribution can be controlled by the internal architecture of a 3D scaffold and that there is a more homogeneous cell distribution in the gradient scaffolds produced in this work remains valid for the seeding technique used. For homogeneous scaffolds (Figs. 12 and 13) the results in terms of cell seeding efficiency and cell distribution are not as good. These scaffolds do not possess any offset and so the cells tend to fall to the bottom of the scaffold, where they mostly attach. We also assume that in both homogeneous scaffolds the flow of medium when adding a cell solution is greater than in the gradient scaffolds, due to the nonexistence of offset layers.

\section{Conclusions}

Application of RP techniques to the production of anatomically adapted bone tissue engineering scaffolds is the only method available that can create predetermined forms and internal architec- 
tures. This work has shown that manipulation of the mechanical properties and some biological parameters can be achieved by optimizing such architectures. Furthermore, starch-based scaffolds could be produced with predefined pore size gradients by a single step fabrication process. In this context, the architecture of a scaffold determines not only the physical properties, but also the cell seeding efficiency and cell distribution within the scaffold. The development of continuous pore size gradients is able to improve cell seeding efficiency and induce a more uniform distribution of cells through the scaffold.

\section{Acknowledgement}

This work was supported by the European NoE EXPERTISSUES (NMP3-CT-2004-500283).

\section{Appendix A}

Figures with essential colour discrimination. Certain figures in this article, particularly Figs. 11-13, are difficult to interpret in black and white. The full colour images can be found in the on-line version, at doi:.10.1016/j.actbio.2010.11.003.

\section{References}

[1] Langer R, Vacanti JP. Tissue engineering. Science 1993;260:920-6.

[2] Flemming RG et al. Effects of synthetic micro- and nano-structured surfaces on cell behavior. Biomaterials 1999;20:573-88.

[3] Desai TA. Micro-and nanoscale structures for tissue engineering constructs. Med Eng Phys 2000;22:595-606.

[4] Andersson H, Van den Berg A. Microfabrication and microfluidics for tissue engineering: state of the art and future opportunities. Lab Chip 2004;4:98-103.

[5] Yeong WY et al. Rapid prototyping in tissue engineering: challenges and potential. Trends Biotechnol 2004;22:643-52.

[6] Lee J et al. Three-dimensional cell culture matrices: state of the art. Tissue Eng Part B Rev 2008;14:61-86.

[7] Hutmacher DW et al. Scaffold-based tissue engineering: Rationale for computer-aided design and solid free-form fabrication systems. Trends Biotechnol 2004;22:354-62.

[8] Hutmacher DW, Cool S. Concepts of scaffold-based tissue engineering-the rationale to use solid free-form fabrication techniques. J Cell Mol Med 2007;11:654-69.

[9] Hutmacher DW. Scaffolds in tissue engineering bone, cartilage. Biomaterials 2000;21:2529-43.

[10] Zein I et al. Fused deposition modeling of novel scaffold architectures for tissue engineering applications. Biomaterials 2002;23:1169-85.

[11] Leong KF et al. Solid freeform fabrication of three-dimensional scaffolds for engineering replacement tissues and organs. Biomaterials 2003:24:2363-78.

[12] Woodfield TBF et al. Design of porous scaffolds for cartilage tissue engineering using a three-dimensional fiber-deposition technique. Biomaterials 2004;25:4149-61.

[13] Malda J et al. The effect of PEGT/PBT scaffold architecture on the composition of tissue engineered cartilage. Biomaterials 2005;26:63-72.

[14] Miot S et al. Effects of scaffold composition and architecture on human nasal chondrocyte redifferentiation and cartilaginous matrix deposition. Biomaterials 2005;26:2479-89.

[15] Moroni L et al. 3D fiber-deposited scaffolds for tissue engineering: Influence of pores geometry and architecture on dynamic mechanical properties. Biomaterials 2006:27:974-85.

[16] Yousefi AM et al. Design and fabrication of 3D-plotted polymeric scaffolds in functional tissue engineering. Polym Eng Sci 2007;47:608-18.

[17] Fedorovich NE et al. Three-dimensional fiber deposition of cell-laden, viable patterned constructs for bone tissue printing. Tissue Eng Part A 2008;14:127-33.

[18] Pfister A et al. Biofunctional rapid prototyping for tissue-engineering applications: 3D bioplotting versus 3D printing. J Polym Sci Part A: Polym Chem 2004;42:624-38.

[19] Kim BS et al. Optimizing seeding and culture methods to engineer smooth muscle tissue on biodegradable polymer matrices. Biotechnol Bioeng 1998;57:46-54

[20] Carrier RL et al. Cardiac tissue engineering: cell seeding, cultivation parameters, and tissue construct characterization. Biotechnol Bioeng 1999;64:580-9.

[21] Xiao YL et al. Static and dynamic fibroblast seeding and cultivation in porous PEO/PBT scaffolds. J Mater Sci: Mater Med 1999;10:773-7.
[22] Holy CE et al. Engineering three-dimensional bone tissue in vitro using biodegradable scaffolds: investigating initial cell-seeding density and culture period. J Biomed Mater Res 2000;51:376-82.

[23] Li Y et al. Effects of filtration seeding on cell density, spatial distribution, and proliferation in nonwoven fibrous matrices. Biotechnol Prog 2001:17:935-44.

[24] Vunjak-Novakovic G, Radisic M. Cell seeding of polymer scaffolds. Methods Mol Biol 2004;238:131-46.

[25] Galban CJ, Locke BR. Effects of spatial variation of cells, nutrient, product concentrations coupled with product inhibition on cell growth in a polymer scaffold. Biotechnol Bioeng 1999;64:633-43.

[26] Yilgor P et al. 3D plotted PCL scaffolds for stem cell based bone tissue engineering. Macromol Symp 2008;269:92-9.

[27] El-Ayoubi R et al. Design and fabrication of 3D porous scaffolds to facilitate cell-based gene therapy. Tissue Eng Part A 2008;14:1037-48.

[28] Li JP et al. Bone ingrowth in porous titanium implants produced by 3D fiber deposition. Biomaterials 2007;28:2810-20.

[29] Moroni L et al. Anatomical 3D fiber-deposited scaffolds for tissue engineering: designing a neotrachea. Tissue Eng 2007;13:2483-93.

[30] Detsch R et al. 3D-cultivation of bone marrow stromal cells on hydroxyapatite scaffolds fabricated by dispense-plotting and negative mould technique. J Mater Sci: Mater Med 2008;19:1491-6.

[31] Moroni L., et al. 3D fiber-deposited electrospun integrated scaffolds enhance cartilage tissue formation. Adv Funct Mater. 2008;18 11:53-60.

[32] Woodfield TBF et al. Polymer scaffolds fabricated with pore-size gradients as a model for studying the zonal organization within tissue-engineered cartilage constructs. Tissue Eng 2005;11:1297-311.

[33] Harley BA et al. Fabricating tubular scaffolds with a radial pore size gradient by a spinning technique. Biomaterials 2006;27:866-74.

[34] Silva MMCG et al. The effect of anisotropic architecture on cell and tissue infiltration into tissue engineering scaffolds. Biomaterials 2006;27:5909-17.

[35] Oh SH et al. In vitro and in vivo characteristics of PCL scaffolds with pore size gradient fabricated by a centrifugation method. Biomaterials 2007;28:1664-71.

[36] Kim G et al. Hybrid process for fabricating 3D hierarchical scaffolds combining rapid prototyping and electrospinning. Macromol Rapid Commun 2008;29:1577-81.

[37] Martins A et al. Hierarchical starch-based fibrous scaffold for bone tissue engineering applications. J Tissue Eng Regener Med 2009;3:37-42.

[38] Kubo K et al. Cellular behavior on $\mathrm{TiO}_{2}$ nanonodular structures in a micro-tonanoscale hierarchy model. Biomaterials 2009;30:5319-29.

[39] Park S et al. 3D polycaprolactone scaffolds with controlled pore structure using a rapid prototyping system. J Mater Sci: Mater Med 2009;20:229-34.

[40] Marques AP et al. Effect of starch-based biomaterials on the in vitro proliferation and viability of osteoblast-like cells. J Mater Sci: Mater Med 2005; $16: 833-42$

[41] Li WJ et al. Biological response of chondrocytes cultured in three-dimensional nanofibrous poly(epsilon-caprolactone) scaffolds. J Biomed Mater Res, Part A 2003;67:1105-14.

[42] Alves CM et al. The dynamics, kinetics and reversibility of protein adsorption onto the surface of biodegradable materials. Soft Matter 2010;6:4135-43.

[43] Marques AP et al. An in vivo study of the host response to starch-based polymers and composites subcutaneously implanted in rats. Macromol Biosci 2005;5:775-85.

[44] Vehof JWM et al. Bone formation in CaP-coated and noncoated titanium fiber mesh. J Biomed Mater Res, Part A 2003;64:417-26.

[45] Landers R, Mulhaupt R. Desktop manufacturing of complex objects, prototypes and biomedical scaffolds by means of computer-assisted design combined with computer-guided 3D plotting of polymers and reactive oligomers. Macromol Mater Eng 2000;282:17-21.

[46] Landers $\mathrm{R}$ et al. Rapid prototyping of scaffolds derived from thermoreversible hydrogels and tailored for applications in tissue engineering. Biomaterials 2002;23:4437-47.

[47] Meyle J et al. Fibroblast anchorage to microtextured surfaces. J Biomed Mater Res 1993;27:1553-7.

[48] Liu XM et al. Influence of substratum surface chemistry/energy and topography on the human fetal osteoblastic cell line hFOB 1.19: phenotypic and genotypic responses observed in vitro. Biomaterials 2007;28:4535-50.

[49] Marcotte L, Tabrizian A. Sensing Surfaces: challenges in studying the cell adhesion process and the cell adhesion forces on biomaterials. IRBM 2008;29:77-88.

[50] Bretcanu O et al. Simple methods to fabricate Bioglass (R)-derived glassceramic scaffolds exhibiting porosity gradient. J Mater Sci 2008;43:4127-34.

[51] Tamayo A et al. Gradient pore size distributions in porous silicon oxycarbide materials. J Eur Ceram Soc 2008;28:1871-9.

[52] $\mathrm{Wu} \mathrm{H}$ et al. Fabrication of chitosan-g-polycaprolactone copolymer scaffolds with gradient porous microstructures. Mater Lett 2008;62:2733-6.

[53] Alves NM et al. Microhardness of starch based biomaterials in simulated physiological conditions. Acta Biomater 2007;3:69-76.

[54] Hutmacher DW et al. Mechanical properties and cell cultural response of polycaprolactone scaffolds designed and fabricated via fused deposition modeling. J Biomed Mater Res 2001;55:203-16.

[55] Guarino V et al. Porosity and mechanical properties relationship in PCL porous scaffolds. J Appl Biomater Biomech 2007;5:149-57.

[56] Yu $\mathrm{H}$ et al. Effect of porosity and pore size on microstructures and mechanical properties of poly-epsilon-caprolactone-hydroxyapatite composites. J Biomed Mater Res B Appl Biomater 2008;86:541-7. 
1018

J.M. Sobral et al./Acta Biomaterialia 7 (2011) 1009-1018

[57] Sen A et al. Creep in injection molded starch/synthetic polymer blends. Mater Sci Eng, A 2002;338:60-9.

[58] Odusanya OS et al. Effect of starch predrying on the mechanical properties of starch/poly(epsilon-caprolactone) composites. J Apple Poly Sci 2003;87:877-84.

[59] Preechawong D et al. Characterization of starch/poly(epsilon-caprolactone) hybrid foams. Polym Test 2004;23:651-7.

[60] Gores ME et al. Starch-poly(epsilon-caprolactone) and starch-poly(lactic acid) fibre-mesh scaffolds for bone tissue engineering applications: structure, Mechanical properties and degradation behaviour. J Tissue Eng Regener Med 2008;2:243-52.

[61] Mono JF et al. Dynamic mechanical properties of hydroxyapatite-reinforced and porous starch-based degradable biomaterials. J Mater Sci: Mater Med 1999;10:857-62.

[62] Mono JF et al. Effects of moisture and degradation time over the mechanical dynamical performance of starch-based biomaterials. J App Polym Sci 2000;78:2345-57.

[63] Espigares I et al. New partially degradable and bioactive acrylic bone cements based on starch blends and ceramic fillers. Biomaterials 2002;23:1883-95.

[64] Mano JF, Reis RL. Viscoelastic monitoring of starch-based biomaterials in simulated physiological conditions. Mater Sci Eng, A 2004;370:321-5.

[65] Gosh S et al. Dynamic mechanical behavior of starch-based scaffolds in dry and physiologically simulated conditions: effect of porosity and pore size. Acta Biomater 2008;4:950-9.

[66] Sousa RA et al. Mechanical performance of starch based bioactive composite biomaterials molded with preferred orientation. Poly Eng Sci 2002;42:1032-45.
[67] Mano JF et al. Thermal properties of thermoplastic starch/synthetic polymer blends with potential biomedical applicability. J Mater Sci: Mater Med 2003;14:127-35.

[68] Elvira C et al. Starch-based biodegradable hydrogels with potential biomedical applications as drug delivery systems. Biomaterials 2002;23:1955-66.

[69] Buechner PM et al. A broadband viscoelastic spectroscopic study of bovine bone: implications for fluid flow. Ann Boomed Eng 2001;29:719-28.

[70] Hsu SH et al. Evaluation of the growth of chondrocytes and osteoblasts seeded into precision scaffolds fabricated by fused deposition manufacturing. J Boomed Mater Res B Appl Biomater 2007;80:519-27.

[71] Mooney DJ, Langer RS. Engineering biomaterials for tissue engineering The 10100 micron size scale. In: Bronzino JD, editor. The biomedical engineering handbook. Bock Ration, FL: CRC Press; 2000.

[72] Karageorgiou V, Kaplan D. Porosity of 3D biomaterial scaffolds and osteogenesis. Biomaterials 2005;26:5474-91.

[73] Uebersax L et al. Effect of scaffold design on bone morphology in vito. Tissue Eng 2006;12:3417-29.

[74] Went D et al. Oscillating perfusion of cell suspensions through threedimensional scaffolds enhances cell seeding efficiency and uniformity. Biotechnol Bioeng 2003;84:205-14.

[75] Du DJ et al. Oscillatory perfusion seeding and culturing of osteoblast-like cells on porous beta-tricalcium phosphate scaffolds. J Biomed Mater Res, Part A 2008;86:796-803. 